\title{
Use of a Yeast tRNase Killer Toxin to Diagnose Kti12 Motifs Required for tRNA Modification by Elongator
}

\author{
Constance Mehlgarten ${ }^{1}$, Heike Prochaska ${ }^{1}$, Alexander Hammermeister ${ }^{2}$, Wael Abdel-Fattah ${ }^{2}$ (iD, \\ Melanie Wagner ${ }^{1}$, Rościsław Krutyhołowa ${ }^{3,4}$ iD, Sang Eun Jun ${ }^{5}$, Gyung-Tae Kim ${ }^{5}$, \\ Sebastian Glatt $^{3}$ (D), Karin D. Breunig ${ }^{1}$, Michael J. R. Stark ${ }^{6}$ (D) and Raffael Schaffrath ${ }^{1,2, *}$ \\ 1 Institut für Biologie, Martin Luther Universität Halle-Wittenberg, Weinbergweg 10, 06120 Halle/Saale, \\ Germany; constance.mehlgarten@genetik.uni-halle.de (C.M.); h.prochaska@nomadbioscience.de (H.P.); \\ Melanie.Wagner@oncotec.de (M.W.); karin.breunig@genetik.uni-halle.de (K.D.B.) \\ 2 Institut für Biologie, FG Mikrobiologie, Universität Kassel, Heirich-Plett-Str. 40, 34132 Kassel, Germany; \\ alex_hammermeister@t-online.de (A.H.); wael@uni-kassel.de (W.A.-F.) \\ 3 Max Planck Research Group at the Malopolska Centre of Biotechnology, Jagiellonian University, \\ 31-007 Krakow, Poland; rostyslav.krutyholova@uj.edu.pl (R.K.); sebastian.glatt@uj.edu.pl (S.G.) \\ 4 Department of Cell Biochemistry, Faculty of Biochemistry, Biophysics and Biotechnology, \\ Jagiellonian University, 31-007 Krakow, Poland \\ 5 Department of Molecular Biotechnology, Dong-A University, Busan 604-714, Korea; \\ junse033@hanmail.net (S.E.J.); kimgt@donga.ac.kr (G.-T.K.) \\ 6 Centre for Gene Regulation \& Expression, School of Life Sciences, University of Dundee, Dundee DD1 5EH, \\ UK; m.j.r.stark@dundee.ac.uk \\ * Correspondence: schaffrath@uni-kassel.de; Tel.: +49-561-804-4175; Fax: +49-561-804-4337
}

Academic Editor: Manfred J. Schmitt

Received: 31 July 2017; Accepted: 3 September 2017; Published: 5 September 2017

\begin{abstract}
Saccharomyces cerevisiae cells are killed by zymocin, a tRNase ribotoxin complex from Kluyveromyces lactis, which cleaves anticodons and inhibits protein synthesis. Zymocin's action requires specific chemical modification of uridine bases in the anticodon wobble position (U34) by the Elongator complex (Elp1-Elp6). Hence, loss of anticodon modification in mutants lacking Elongator or related KTI ( $\underline{K}$. lactis Toxin Insensitive) genes protects against tRNA cleavage and confers resistance to the toxin. Here, we show that zymocin can be used as a tool to genetically analyse KTI12, a gene previously shown to code for an Elongator partner protein. From a kti12 mutant pool of zymocin survivors, we identify motifs in Kti12 that are functionally directly coupled to Elongator activity. In addition, shared requirement of U34 modifications for nonsense and missense tRNA suppression (SUP4; SOE1) strongly suggests that Kti12 and Elongator cooperate to assure proper tRNA functioning. We show that the Kti12 motifs are conserved in plant ortholog DRL1/ELO4 from Arabidopsis thaliana and seem to be involved in binding of cofactors (e.g., nucleotides, calmodulin). Elongator interaction defects triggered by mutations in these motifs correlate with phenotypes typical for loss of U34 modification. Thus, tRNA modification by Elongator appears to require physical contact with Kti12, and our preliminary data suggest that metabolic signals may affect proper communication between them.
\end{abstract}

Keywords: zymocin; ribotoxin; tRNase; Kti12; Elongator complex; tRNA anticodon modification

\section{Introduction}

Zymocin, a composite chitinase and tRNA anticodon nuclease (tRNase) ribotoxin produced from the dairy yeast Kluyveromyces lactis, kills other yeast species including Saccharomyces cerevisiae [1,2]. Although zymocin is a trimeric complex $(\alpha \beta \gamma)$, expression of its $\gamma$-subunit (i.e., $\gamma$-toxin tRNase) alone 
is lethal in $S$. cerevisiae, suggesting that the $\alpha / \beta$-subunits facilitate zymocin docking and $\gamma$-toxin delivery [2,3]. Accordingly, screens for zymocin survivors identified mutations in KTI (K. lactis toxin insensitive) and IKI (insensitive to killer toxin) genes that fall into non-target (class I) and toxin-target (class II) groups based on their response to conditional $\gamma$-toxin induction [3-5]. Class I genes operate in the synthesis of cell wall and membrane components (chitin, sphingolipids, $\mathrm{H}^{+}$pump Pma1) required for zymocin binding and $\gamma$-toxin entry, and class II loci identified a toxin-target effector role for the Elongator complex (Elp1-Elp6) [2,6]. Originally, the latter had been co-purified with elongating RNA polymerase II, hence its name [7]. However, more recently a robust body of evidence indicates that its genuine role in yeast lies with tRNA modification rather than transcription elongation. Accordingly, Elongator binds tRNAs and modifies anticodon wobble uridine (U34) bases [8-14]. Strikingly, the methoxy-carbonyl-methyl-thio $\left(\mathrm{mcm}^{5} \mathrm{~s}^{2}\right)$ modification at U34 in some of Elongator's tRNA substrates (including tRNA ${ }^{\text {Glu}}$ ) allows anticodon cleavage by the $\gamma$-toxin tRNase [15]. Therefore, loss of U34 modification in Elongator mutants protects efficiently against the toxin's attack $[6,15,16]$.

Elevated tRNA ${ }^{\text {Glu }}$ levels also protect against zymocin, and this effect is suppressed by overexpression of the class II gene KTI1/TRM9 [15,16]. Trm9 is the catalytic subunit of a methyl-transferase (Trm9•Trm112), which methylates the preceding $\mathrm{cm}^{5}\left(\mathrm{~s}^{2}\right) \mathrm{U} 34$ modification generated by Elongator and also contributes to zymocin sensitivity [16-18]. Consistently, loss of U34 methylation in trm 9 and trm112 mutants confers resistance to zymocin and $\gamma$-toxin tRNase [18,19]. Thus, growth inhibition of $S$. cerevisiae by zymocin apparently involves restriction of U34-modified tRNAs affecting protein biosynthesis. In support of this strategy, which is shared by other microbial anticodon nucleases [20,21], zymocin depletes tRNA ${ }^{\text {Glu }}$ in vivo, and overexpression of tRNA ligase suppresses the tRNA attack showing that tRNA repair is able to 'heal' the damage in the anticodon loop [22]. Use of the $\gamma$-toxin tRNase as a tool diagnostic for Elongator activity identified additional class II gene products with related roles in tRNA modification [2,5,19,23-25]. Among them, a casein kinase I isozyme (Hrr25/Kti14), a protein phosphatase (Sit4) and an Elongator partner protein (Kti12) were found to constitute a complicated network that regulates the phosphorylation status of Elongator's largest subunit (Elp1) [26-28]. This suggested that phosphorylation affects Elongator activity, a notion supported by data showing that Hrr 25 phosphorylation sites on Elp1 are critical for U34 modification [29] and that Hrr25 association with Elongator requires Kti12 [28].

Sequence alignments between Kti12 and its plant ortholog DRL1/ELO4 suggested that both share a P-loop motif characteristic for nucleoside triphosphate (NTP) binders [30] and a putative calmodulin (CaM) binding domain (CBD) [31]. With ELO4 known to bind CaM in vitro and associate with plant Elongator in vivo [32,33], the presence of cofactor binding motifs points towards conserved roles for Kti12 and ELO4. To further analyze Kti12 requirements for Elongator activity, we studied a pool of zymocin resistant kti12 mutants previously used [5] to identify the KTI12 locus in yeast. We map mutations in the CBD and P-loop motifs and other regions conserved between Kti12 and ELO4 that, based on genetic and biochemical assays, trigger traits typical of Elongator-linked loss of U34 modification and defects in Elongator interaction. In summary, our data reinforce the view that Kti12 relates in function to the yeast Elongator complex and stimulates its tRNA modification activity.

\section{Results and Discussion}

Based on its similarity to yeast Kti12, the Arabidopsis protein ELO4 was previously proposed to play an Elongator-related role in plants [24,32]. This was confirmed when ELO4 was shown to associate with TAP-tagged plant Elongator and ELO4 mutations found to cause phenotypes typical of plant Elongator mutants [33]. In addition, alignments between Kti12 and ELO4 proteins from fungal and plant species (Figure S1) revealed several regions of high similarity. Among these are P-loop motifs, putative calmodulin (CaM) binding domains (CBDs) [24,31,32] and other homology regions (Figure S1). While P-loops may be indicative of NTP binding [30], the CBDs likely mediate the demonstrated in vitro binding of ELO4 to CaM [31,32]. In comparison to strong CBDs (type 1-5-10) used, e.g., in tandem affinity purification (TAP) protocols [34], the CBDs in Kti12 or ELO4 (type 1-8-14) 
show weaker CaM affinity [35]. In summary, similarity in sequence and domain organisation may indicate that the motifs found in Kti12 and ELO4 are evolutionarily conserved because they functionally interact with Elongator.

\subsection{A kti12 Mutant Pool Reveals Conserved Kti12 Motifs with Elongator-Related Roles}

Given that KTI12 deletions cause Elongator defects [6,24,36], we analysed a previously isolated pool of zymocin resistant $k t i 12$ mutants [5] in greater detail (Figure 1A). Single amino acid substitutions within full-length Kti12 were mapped to the P-loop ( $k$ ti12-4: S12R) and CBD1 (kti12-8: G92D) motifs (Figure 1A). In addition, nonsense (kti12-1, kti12-2, kti12-3 and kti12-6) or frame-shift (kti12-5 and kti12-7) mutations led to truncations resulting in deletion of various invariant Kti12 regions including one or both CBD motifs (Figure 1A). Following assays diagnostic for Elongator-minus phenotypes, all $k t i 12$ mutants were found to be zymocin resistant, hypersensitive to $7.5 \mathrm{mM}$ caffeine (data not shown) and sensitive to temperatures above $38^{\circ} \mathrm{C}$ (Figure 1B). Collectively, these traits phenocopy kti12 $\Delta$ and elp $3 \Delta$ strains (Figure 1B), reinforcing the view that Kti12 and Elongator are closely related in function $[6,24,36]$.

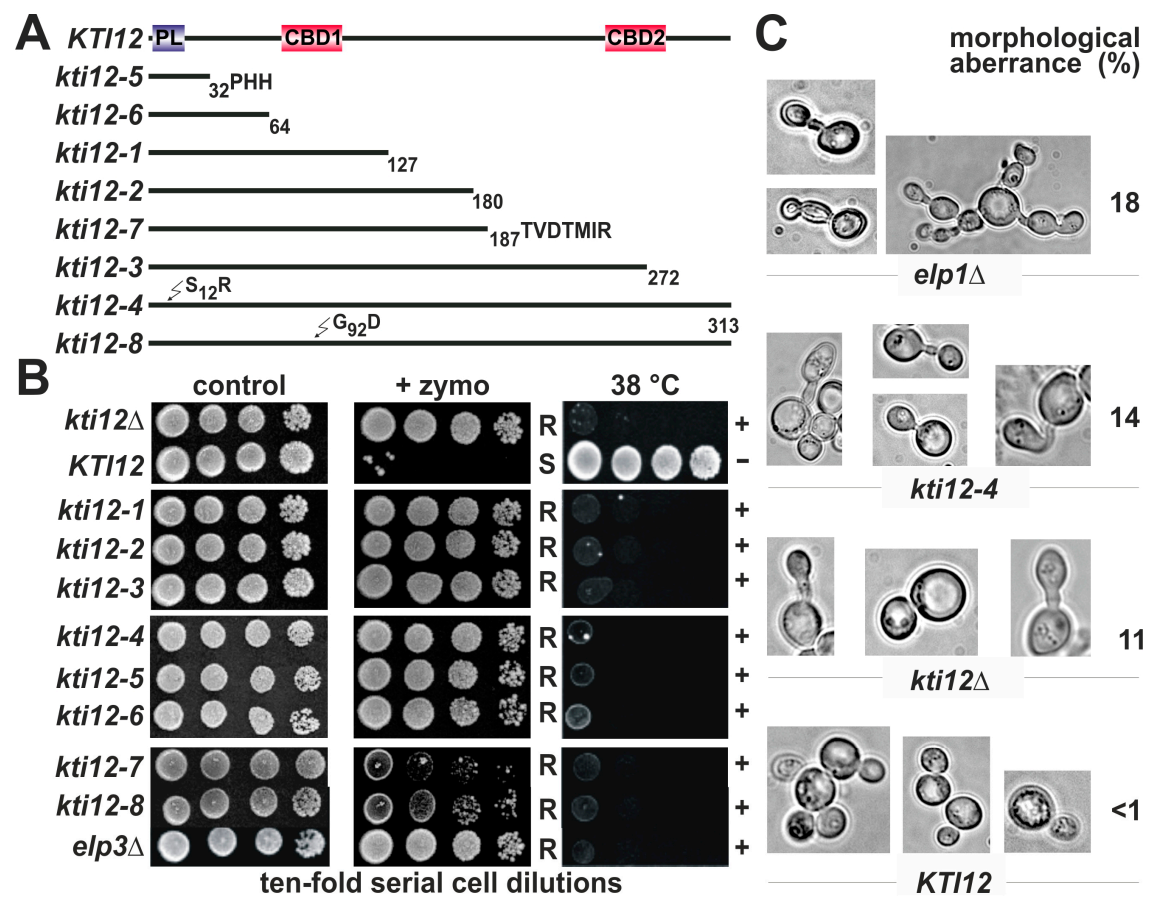

Figure 1. A set of kti12 mutants uncovers a phenotypic signature typical of Elongator defects. (A) compilation of kti12 mutants including mutation mapping and alignment to Kti12 domain organization (for details, see Figure S1); (B,C) phenotypes shared between kti12 and Elongator mutants. Growth assays (B) with yeast strains of the indicated backgrounds at $30{ }^{\circ} \mathrm{C}$ (control), elevated temperature $\left(38{ }^{\circ} \mathrm{C}\right)$ or in the presence of $45 \%$ [v/v] zymocin (+zymo). Traits typical of Elongator mutant (elp3 $\Delta$ ) include zymocin resistance (R) and thermosensitivity (+); zymocin sensitivity (S) and thermotolerance (-) denote wild-type (KTI12) phenotypes; (C) phase contrast microscopy to monitor cell/bud morphology in the indicated strain backgrounds.

In addition, significant proportions of $k t i 12 \Delta(11 \%), k t i 12-4(14 \%)$ and $\operatorname{elp} 1 \Delta(14 \%)$ mutants showed aberrantly elongated cells/buds (Figure 1C), morphological defects previously observed in association with Elongator or related tRNA modification mutants including $k t i 14 / h r r 25$ cells $[25,28,37]$. Consistent with Elongator mutations known to suppress thermosensitive growth of $\sec 2-59^{\text {ts }}$ and $\sec 12-4^{\text {ts }}$ mutants at $31^{\circ} \mathrm{C}$ and $33{ }^{\circ} \mathrm{C}$, respectively [38,39], sec mutant rescue was also seen with the kti12-4 and kti12-8 alleles (Figure S2), although to a lesser extent than elp1 $\Delta$. In summary, an Elongator-like phenotypic 
signature of KTI12 loss-of-function alleles documents the usefulness of zymocin as a tool diagnostic for Kti12 protein motifs that are important for Elongator activity.

\subsection{Conserved Kti12 Motifs Support Elongator-Dependent tRNA Suppression}

Physiologically, Elongator-dependent tRNA modification fine-tunes tRNA decoding [40-43] and maintains ribosomal reading frame accuracy during translation [44-47]. In support, stop codon read-through by U34 carrying tRNA suppressors (SUP4; sup9e) requires Elongator function [8,16,39]. Inspired by previous data showing that some class II $k t i$ mutants also have tRNA modification defects $[8,19]$, we compared SUP4 read-through between members of the kti12 mutant collection and an elp3 $\Delta$ knock-out in reporter strain UMY2893. The latter maintains ochre (UAA) alleles of ADE2 (ade2-1) and CAN1 (can1-100), which upon suppression by SUP4, confer adenine prototrophy $\left(\mathrm{Ade}^{+}\right)$and canavanine sensitivity $\left(\mathrm{Can}^{\mathrm{S}}\right)$, respectively $[8,16]$. In agreement with previous reports, SUP4 read-through of ade2-1 and can1-100 was abolished by ELP3 gene deletion yielding adenine auxotrophic $\left(\mathrm{Ade}^{-}\right)$and canavanine resistant $\left(\mathrm{Can}^{\mathrm{R}}\right)$ elp $3 \Delta$ cells (Figure 2A). Notably, both traits (Ade ${ }^{-}$, $\mathrm{Can}^{\mathrm{R}}$ ) were copied by all the $k t i 12$ mutant alleles (kti12-1, kti12-3, kti12-4, kti12-6, kti12-7 and kti12-8) we tested (Figure 2A) and complemented after reintroducing a KTI12 wild-type gene into kti12 $\Delta$ cells (Figure 2A). The latter finding supports our notion that recessive KTI12 loss-of-function alleles trigger Elongator-linked tRNA modification defects and phenotypes in yeast (Figures 1 and 2).
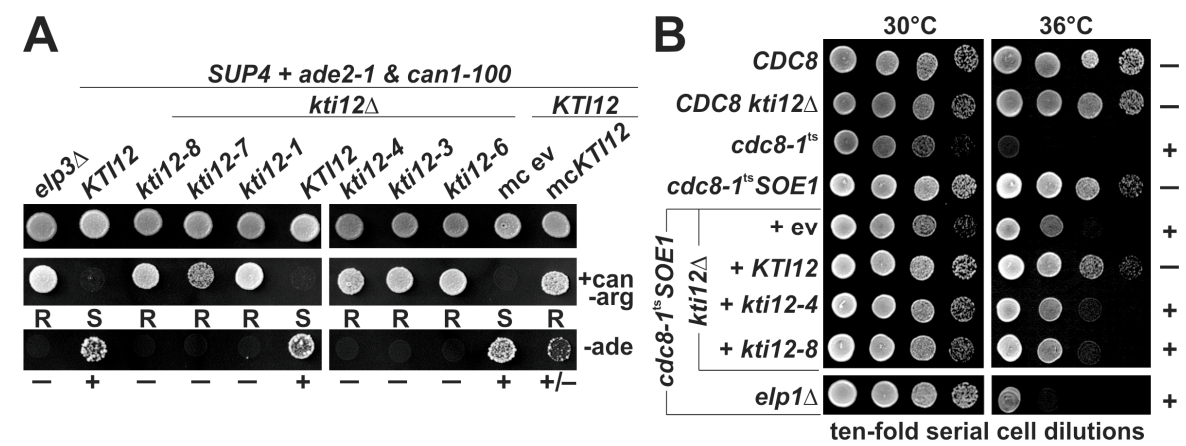

Figure 2. KTI12 gene mutations and copy number interfere with U34 tRNA suppressors. (A) tRNA nonsense suppression. In the indicated genetic backgrounds, can1-100 and ade2-1 ochre read-through by SUP4 was phenotypically assessed by canavanine sensitivity/resistance (S/R: middle panel) on canavanine supplemented medium lacking arginine (+can - arg) or by adenine proto-/auxotrophy (+/ - : bottom panel) on minimal medium without adenine (-ade). Growth control (top panel) involved yeast peptone dextrose (YPD) rich medium. Empty vector (ev) served as reference for multi-copy (mc) KTI12; (B) SOE1 tRNA missense suppression. The indicated strains were grown at temperatures permissive $\left(30{ }^{\circ} \mathrm{C}\right)$ or restrictive $\left(36^{\circ} \mathrm{C}\right)$ for $c d c 8-1^{\text {ts }}$ cells. Note that ELP1 and KTI12 gene mutations affect $(+)$ rescue of $c d c 8-1^{\text {ts }}$ cell growth at $36^{\circ} \mathrm{C}(-)$ by the tRNA suppressor SOE1.

Consistent with our previous observation that Elongator dependent processes (including e.g., zymocicity) can be suppressed by overexpression of KTI12 [5,26], multi-copy KTI12 also countered tRNA suppression of can1-100 by SUP4 (Figure 2A). As for ade2-1, however, multi-copy KTI12, allowed weak growth in medium lacking adenine, a trait in between the phenotypes of wild-type (Ade ${ }^{+}$) or elp3/kti12 mutant ( $\mathrm{Ade}^{-}$) cells (Figure 2A). The latter read-out may indicate that the two ochre reporters differ in their level of sensitivity level to Elongator defects. Presumably, a weaker Ade ${ }^{+}$trait could be interpreted in a way that increased intracellular Kti12 levels also decrease the tRNA modification activity of Elongator (Figure 2A). It will be interesting to address this scenario in greater detail in future studies using quantitative lacZ ${ }^{\text {ochre }}$ read-through assay or direct LC-MS/MS measurements $[29,48,49]$. These techniques will allow comparison of the relative abundance of Elongator dependent U34 modification levels between mutants lacking Kti12 and cells expressing Kti12 at elevated levels from either single-copy or multi-copy plasmids. 
In addition, we observed that similar to $k t i 12 \Delta$ and elp1 $\Delta$ cells, kti12-4 and kti12-8 alleles also antagonized tRNA missense suppression of $c d c 8-1^{\text {ts }}$ by SOE1 (Figure 2B), a mutant tRNA Glu that reads Lys codons in a fashion dependent on U34 modification by Elongator [39,50]. Hence, proper Elongator and Kti12 activity are required for SOE1 to rectify Glu to Lys substitutions in $c d c 8-1^{\text {ts }}$ cells and thus suppress their thermosensitivity at $36{ }^{\circ} \mathrm{C}$ (Figure 2B). Together with previous data showing that missense (SOE1) and nonsense (SUP4; sup9e) suppressor tRNAs depend on Elongator [8,16,39], Kti12 can thus be considered to promote suppressor tRNA functionality, most likely through its regulatory role in Elongator's tRNA modification reaction.

\subsection{Kti12 Expression and Interaction Profiles}

To examine whether and how the mutated Kti12 proteins are expressed, we subjected kti12-2, kti12-3, kti12-4 and kti12-8 alleles to genomic c-Myc-tagging. Following Western blot analyses, all epitope-tagged Kti12 variants were found to be produced at the expected molecular weights (Figure 3A); Kti12-4 and Kti12-8 are full-length ( 40 kDa) while Kti12-2 $(\sim 26 \mathrm{kDa})$ and Kti12-3 $(\sim 35 \mathrm{kDa})$ represent C-terminal truncations (Figure 3A). In addition, while Kti12-3 and Kti12-4 levels were reduced, Kti12-2 and Kti12-8 expression compared favourably with Kti12 wild-type levels (Figure 3A). To avoid abundance related secondary effects, we conducted subsequent analyses with the latter two mutants. Since Kti12 and Elongator associate with each other $[6,24,28,36]$, we examined whether inappropriate interaction between both may underlie the loss-of-function traits observed in kti12-2 and kti12-8 cells (Figures 1 and 2). Therefore, c-Myc-tagged KTI12, kti12-2 and kti12-8 alleles (Figure 3A) were each co-expressed with an HA-tagged copy of the ELP2 gene for co-immune precipitation (IP) assays (Figure 3B). Unlike the strong interaction observed between wild-type Kti12 and Elp2 (Figure 3B), the Elongator subunit failed to co-precipitate Kti12-2 and Kti12-8 (Figure 3B) and both mutants hardly co-purified Elp2 in reciprocal IPs (data not shown). This indicates that a proper interaction with Elongator requires both the structural integrity of CBD2 and conserved Kti12 residues further downstream (truncated in kti12-2 cells) and an intact CBD1 motif (mutated in kti12-8 cells) (Figure 1A). Whether the G92D mutation (Figure 1A) affects Elongator interaction in kti12-8 cells through changes in local hydrophobicity (Figure S4) is an option currently under investigation. In summary, loss of Elongator binding due to mutations in specific and putative functional Kti12 domains correlates with Elongator-minus traits (Figures 1 and 2). These observations again support our previously presented working hypothesis that direct physical contact with Kti12 is required for Elongator's ability to modify tRNA anticodons $[6,28,29]$.

A

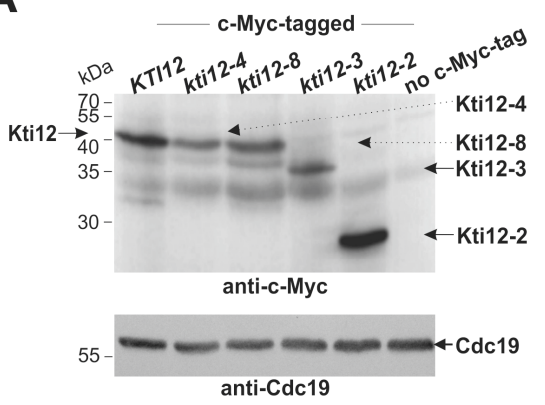

B

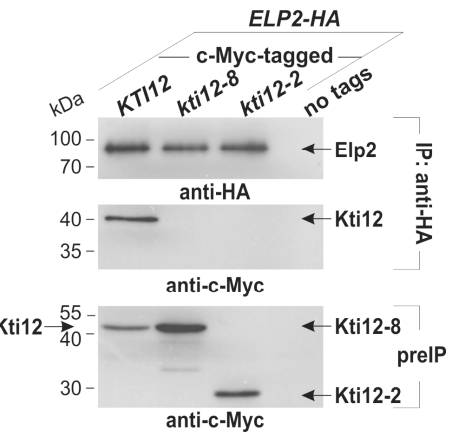

Figure 3. Kti12 expression and Elongator interaction studies. (A) expression of Kti12 and its mutated variants. The indicated KTI12/kti12 alleles were genomically c-Myc-tagged and each gene product identified (top panel) in anti-c-Myc Western blots of total yeast protein extracts. An independent, anti-Cdc19 blot served as internal control (bottom panel); (B) elongator interaction studies with Kti12 and its mutants. Strains co-expressing Elp2-HA and c-Myc-tagged Kti12, Kti12-2 or Kti12-8 were subjected to anti-HA immune precipitation (IP). The IPs were probed for Elp2-HA (top panel) or Kti12-c-Myc (middle panel) with anti-HA or anti-c-Myc antibodies, respectively. Total protein extracts (preIP, bottom panel) probed with anti c-Myc antibodies served as Kti12 input control. 


\subsection{Affinity of Kti12 for CaM}

As the putative CBDs may be involved in Kti12 function (Figures 2 and 3) [24,31,32], we examined next whether yeast Kti12 binds to CaM. In detail, we subjected total yeast extracts containing c-Myc-tagged Kti12 or Kti12-8 to CaM Sepharose affinity chromatography. Extracts containing Kti13-c-Myc, a protein without any CBD motif, or Kti12-TAP with a high-affinity CaM-tag were used as negative and positive controls, respectively (Figure 4). Protein bound to the CaM matrix was eluted with EGTA to chelate calcium and analyzed in Western blots. Both wild-type Kti12-c-Myc and Kti12-8-c-Myc could be detected in the eluate, whereas the negative control Kti13-c-Myc could not (Figure 4). The flow-through fractions indicate that, as expected, the affinity of Kti12-c-Myc is lower than that of Kti12-TAP, which carries an additional CBD derived from the TAP tag [34]. Whether the second CBD (Figure S1) might contribute to residual binding of the kti12-8 mutant to CaM is not known. Collectively, our data indicate that Kti12 and Kti12-8 bind CaM in a calcium sensitive manner. Although they cannot unambiguously provide evidence that the Gly-92 residue in Kti12 is part of a bona fide CBD, they show that Kti12 has CaM affinity, a finding consistent with demonstrated in vitro binding of plant ELO4 to CaM [32]. Whether the ability of Kti12 to bind CaM contributes to Elongator function has to await further investigation.

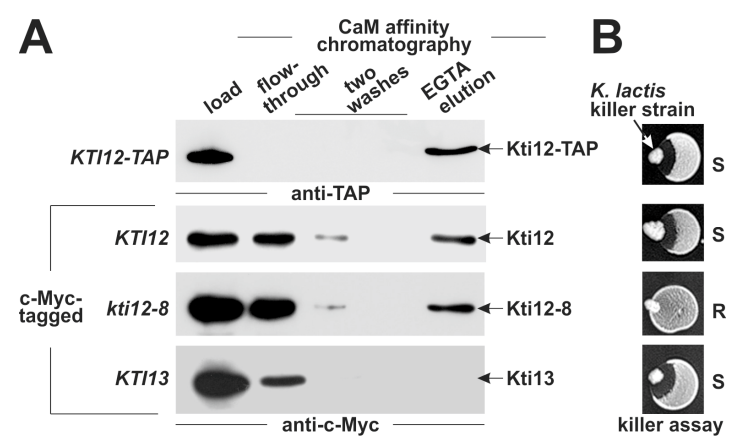

Figure 4. CaM binding by Kti12 in vitro. (A) CaM affinity chromatography of Kti12 and Kti13 variants from the indicated genetic backgrounds. Equal proportions of input (load), unbound (flow-through; washes) and eluted (EGTA elution) protein fractions were subjected to anti-TAP (top panel) and anti c-Myc Western blots (other panels); (B) killer eclipse assays between the same S. cerevisiae strains used for CaM affinity chromatography and a K. lactis zymocin producer (killer strain). ' $\mathrm{S}$ ' or ' $\mathrm{R}$ ' denote zymocin sensitivity or resistance.

\subsection{Cross-Complementation Analysis between KTI12 and ELO4}

Based on several lines of evidence including sequence similarities (27.9\% identity; $45.3 \%$ similarity, Figure S1), elongator-related functions in vivo and CaM binding in vitro (Figure 4) [31-33], Kti12 and ELO4 may be functional counterparts. To study structure and functional conservation between subdomains (Figure 5A), we engineered hybrid alleles of KTI12 encoding motif-swapped proteins in which the P-loop or CBD1 motif from Kti12 is replaced with the respective ELO4 sequence (Figure 5B). After introducing these into a $k t i 12 \Delta$ reporter strain, both hybrids were able to complement the loss-of function phenotype of the mutant; sensitivity to growth inhibition by the $\gamma$-toxin tRNase subunit of zymocin (Figure 5C) and ochre codon read-through of ade2-1 by SUP4 (Figure 5D) were restored. In contrast, the plant ELO4 gene leaves $\gamma$-toxin resistance and adenine auxotrophy (Ade ${ }^{-}$) of $k t i 12 \Delta$ cells unchanged (Figure 5C,D). Hence, the Arabidopsis homologue of Kti12 is unable to replace Kti12 function in yeast, whereas the hybrid proteins are functional. We conclude that sequence differences in the highly conserved motifs are unlikely to be responsible for the specificity of Kti12. Notably, the KTI12 gene from $K$. lactis, which is more closely related to that of S. cerevisiae, is also unable to complement the kti12 $\Delta$ mutation (data not shown). To address the question of whether Elongator interactions are retained in the motif-swapped Kti12-c-Myc variants, they were co-expressed with Elp1-HA, and IPs 
were performed with anti-HA antibodies. Elp1-HA co-precipitated native Kti12 and both hybrids (Figure 5E). Based on protein expression in the extracts prior to IP that were compared to wild-type Kti12 levels, the CBD1-swapped hybrid may be slightly reduced in Elp1 interaction. In summary, we conclude that while alleles coding for hybrids, in which conserved cofactor binding motifs of ELO4 (P-loop, CBD1) have been engineered into yeast Kti12, are functionally exchangeable, full-length plant ELO4 is not. Cross-complementation was also absent with plant ELO4 homologs from rice (OsELO4) and moss (PpELO4) (Figure S3). The incompatibility of ELO4 with Elongator function in S. cerevisiae contrasts with previous findings showing that elp $1 \Delta$ mutants can be partially rescued by $E L O 2$, the plant homolog of yeast ELP1 [51]. Strikingly, the most highly conserved Elongator subunit gene (ELP3) gene cannot be replaced in yeast by the Arabidopsis ELP3 homolog even though the plant subunit assembled into holo-Elongator complexes [9]. However, functional complementation in yeast was achieved when the two plant Elongator genes (ELO2, ELP3) were co-expressed in the genetic background of an elp $1 \Delta$ elp $3 \Delta$ double mutant [9]. Hence, non-conserved plant-specific sequences in ELO4 may mediate a selective physical or functional interaction with individual Elongator subunits, Hrr24 or Sit4 to explain the failure of cross-complementation in our gene shuffle experiments (Figure 5C,D). Regions of species-specific sequence variation may thus provide first insights into the evolutionary differences between plant ELO4 and yeast Kti12.

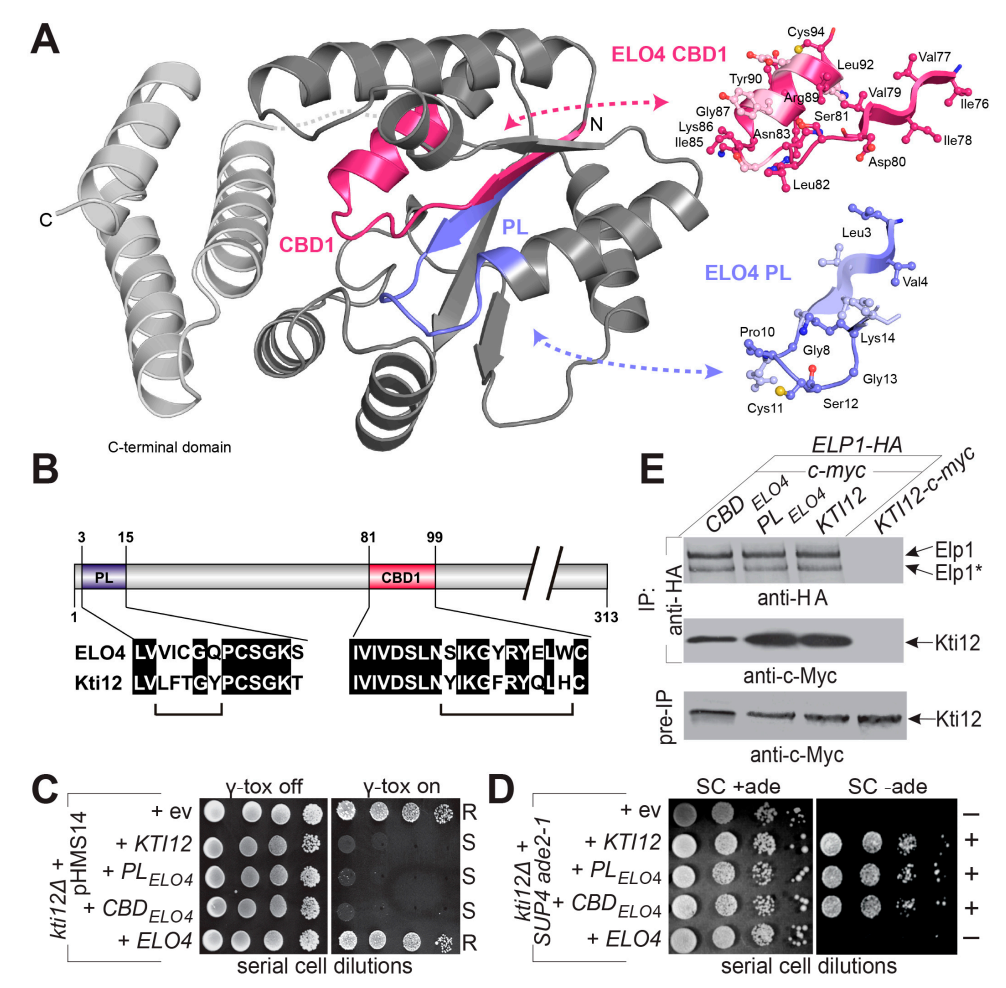

Figure 5. KTI12 and ELO4 gene shuffle analysis. (A) A PSTK homolog-based structural model of yeast Kti12 with highlighted conserved P-loop (blue) and CBD1 (pink) in cartoon representation. PSTK based structures of P-loop (PL) and CBD1 from ELO4 provide a structural insight into the motif swap experiment. Highly conserved residues are shown in ball and stick representation, and labelled respectively; (B) Indication of PL and CBD motif shuffling from ELO4 to Kti12; $(\mathbf{C}, \mathbf{D})$ cross-complementation was studied by (C) conditional $\gamma$-toxin tRNase expression [6] on glucose ( $\gamma$-toxin: off) vs. galactose ( $\gamma$-tox: on) and (D) SUP4 nonsense suppression of ade2-1 (see also Figure 2A). R/S: $\gamma$-toxin resistance/sensitivity; +/-: adenine proto-/auxotrophy; (E) elongator interaction. Strains co-expressing Elp1-HA and indicated c-Myc-tagged Kti12 variants were subjected to anti-HA immune precipitation (IP). The IPs were probed with anti-HA and anti-c-Myc antibodies to check for content of Elp1-HA (top panel) and co-precipitated Kti12 material (middle panel). Total extracts (bottom panel) were probed with anti-c-Myc antibodies and served as input (preIP) control. 


\section{Conclusions}

To conclude, the zymocin tRNase ribotoxin can be exploited as a molecular diagnostic tool to assign function to motifs conserved in two Elongator partner proteins, Kti12 and plant ELO4. These motifs are potentially involved in cofactor binding (nucleotide, calmodulin) and when mutated, abolish Elongator interaction and trigger phenotypes typical of tRNA modification defects in known Elongator mutants. Thus, tRNA modification by Elongator appears to require physical contact and proper communication with Kti12, a notion further supported by our findings that Kti12 and Elongator cooperate in proper performance of nonsense and missense tRNA suppressors (SUP4, SOE1). Despite their structural and functional similarities, Kti12 and ELO4 cannot be functionally exchanged between yeast and plant cells. This suggests the occurrence of species-specific barriers or evolutionary diversification of Elongator and its associated regulatory proteins between lower and higher eukaryotes. In summary, we provide further evidence for a dynamic and complicated network underlying potential regulation of the U34 modification pathway. It constitutes regulatory proteins including Kti11/Dph3, Kti12, Kti13/Ats1 and Kti14/Hrr25 [28,29,36,39,52-55] whose associations with the Elongator complex appear to be sensitive to certain metabolic signals. In support of this notion are recent findings showing that loss of U34 modifications cause altered metabolic profiles in Elongator mutants [56].

\section{Materials and Methods}

\subsection{Yeast Strains, Media and General Methods and Plasmid Constructions}

Growth of yeast strains (Table S1) was in routine YPD or SC media [57] for 3 days at $30{ }^{\circ} \mathrm{C}$. Thermosensitivity was assessed by cultivation at elevated $\left(31^{\circ} \mathrm{C}, 33^{\circ} \mathrm{C}, 36^{\circ} \mathrm{C}, 39^{\circ} \mathrm{C}\right)$ temperatures. Caffeine sensitivity involved YPD supplementation with $7.5 \mathrm{mM}$ of the chemical (Sigma Aldrich, Taufkirchen, Germany). Table S2 lists primers used for PCR-based protocols to epitope tag and delete genes and to map kti12 mutations [3,5] by Sanger DNA sequencing. Transformation of yeast cells with PCR products and plasmids (Table S3) was done as described [58]. Monitoring zymocin sensitivity/resistance used direct colony-colony eclipse assays [4] between K. lactis killer strain AWJ137 (Table S1) and S. cerevisiae tester strains or YPD plate assays containing 40-50\% $(v / v)$ partially purified zymocin [26]. Responses towards intracellular expression of zymocin's lethal tRNase subunit ( $\gamma$-toxin) used GAL1- $\gamma$-toxin expression vector pHMS14 as described [6]. ade2-1 and can1-100 ochre read-through by SUP4 and missense suppression of $c d c 8-1^{\text {ts }}$ by SOE1 followed previous protocols $[8,16,50]$ as did sec2-59ts and sec12-4ts suppression assays [38,39]. Construction details of KTI12 hybrid alleles, in which the P-loop and CBD domains were replaced with ELO4 motifs ( $\mathrm{PL}_{E L O 4} \& \mathrm{CBD}_{E L O 4}$; Figure 5B) by two-step fusion PCR, template plasmid pDJ75 and indicated primers (Table S2), can be made available on request. Expression of ELO4/DRL1 cDNAs from Arabidopsis (AtELO4), rice (PpELO4) and moss (PpELO4) under control of the TDH3 promoter utilized single-copy vector pTU1 [31]. For functional analysis, kti12 mutant and KTI12 wild-type alleles were moved following rescue (see below) from the multi-copy backbone of pJHW27 into single-copy vector YCplac33 [59].

\subsection{Functional Analysis of the kti12 Mutant Pool and Genomic Manipulations at the ELP and KTI Loci}

To identify individual KTI12 mutations from the kti12 pool (kti12-1 to kti12-8) (Table S1), ARB(K) member strains [3,5] raised in LL20 and KY117 backgrounds (Table S1) were subjected to gap repair in vivo using plasmids pJHW27 or pDJ40 (Table S3) restricted with NdeI and NcoI [5] or to direct kti12 locus amplification. The latter used genomic DNA for PCR with Pfu polymerase (Fermentas) and primers KTI12-P and KTI12-4 (Table S2). Yeast plasmids rescued in E. coli or generated by sub-cloning the above PCR products in TOPO pCR2.1 (Invitrogen, Thermo-Fisher Scientific, Waltham, MA, USA) were sequenced in reactions containing $4 \mu \mathrm{L}$ of ready reaction premix $(2.5 \times), 2 \mu \mathrm{L}$ of BigDye terminator v1.1 sequencing buffer $(5 \times)$ (Applied Biosystems, Waltham, MA, USA), 200-500 ng of plasmid DNA and 3.2 pmol of sequencing primers KTI12-P and KTI12-1 to KTI12-4 (Table S2) in a final volume of $20 \mu \mathrm{L}$. Following 30 cycles, the PCR reactions were subjected to ethanol precipitations and run 
on an ABI-Prism 377 DNA sequencer (PerkinElmer, Hamburg, Germany). Tagging of KTI12 alleles and Elongator subunit genes (ELP1, ELP2) with c-Myc or HA epitopes essentially followed previous PCR protocols [6,24] using the S2/S3 primer pairs (Table S2) compatible with the pYM template plasmids (Table S3). KTI12-TAP involved the PCR protocol of Puig et al. [34] with template plasmid pBS1479 (Table S3) and up/down-KTI12primers (Table S2). c-Myc tagging of KTI13 was previously described [23]. KTI12, ELP1 and ELP2 gene deletions were created using published [6,24] PCR protocols, primers and marker plasmids (Tables S2 and S3). Gene deletions were physically verified by PCR on genomic DNA preparations using gene-specific diagnostic primers (Table S2) and phenotypically confirmed by zymocin assays (see above).

\subsection{KTI12 Expression Analyses and Elongator or CaM Interaction Studies}

Immune detection of epitope-tagged Kti12 and Elongator subunits and immune precipitations (IPs) from total yeast extracts used anti-c-Myc (9E10) and anti-HA (3F10) antibodies (Roche) as previously described $[6,24]$. Protein loading was checked with anti-Cdc19 serum (a kind donation by Dr J. Thorner, University of California, Berkley, CA, USA), which recognizes pyruvate kinase. CaM binding assays in vitro involved TAP-tagged or c-Myc-marked Kti12 and Kti13 proteins from total yeast extracts $(\sim 2 \mathrm{mg})$ adjusted to $1 \mathrm{mM} \mathrm{CaCl}_{2}$. The material $(2 \mathrm{~mL})$ was mixed with CaM-Sepharose for $30 \mathrm{~min}$ and equilibrated with $2 \mathrm{~mL} \mathrm{CaM}$ binding buffer (CBB) as described [32]. The slurry was packed onto a column, the buffer drained, and the column rinsed twice with 2.5 volumes of CBB. Elution of bound proteins was in EGTA buffer (4 mM Tris-HCl, pH 7.5, $200 \mathrm{mM} \mathrm{NaCl}, 1 \mathrm{mM} \mathrm{MgCl}$, $2 \mathrm{mM}$ EGTA, and $0.1 \mathrm{mM}$ DTT). Equal proportions of load, flow-through, wash, and elution fractions were separated by $15 \%$ SDS-PAGE, and Kti12 material was detected using anti-TAP and anti-c-Myc Western blots.

\subsection{Kti12 Secondary Structure Predictions and Alignments with ELO4/DRL1}

Secondary structure predictions for Kti12 and ELO4 proteins were obtained using the semi-automated homolog-based protein fold recognition server Phyre2.0 [60]. Best matching records, in both cases based on the crystal structure of archaeal $O$-phosphoseryl-tRNA(Sec) kinase (PSTK, PDB: 3A4M) [61], were further used to visualize Kti12 domain architecture and illustrate a motif-swap experiment (Figure 5B). All structures were rendered using PyMOL Molecular Graphics System (http:/ / www.pymol.org/) (Version 1.8 Schrödinger, LLC, New York, NY, USA). Sequences of Kti12 or its homologs from indicated organisms were obtained from NCBI. Multiple alignments were generated using MUSCLE available at EMBL-EBI web page (http:/ / www.ebi.ac.uk/Tools/msa/muscle/). MSA files were handled using JalView software [62]. Homolog-based model and multiple alignment were used for sequence conservation illustration with the CONSURF server (http://consurf.tau.ac.il/) [63].

Supplementary Materials: The following are available online at www.mdpi.com/2072-6651/9/9/272/s1, Figure S1: Multiple Kti12 sequence alignment; Figure S2: ELP1 and KTI12 mutations rescue thermosensitivity of sec2-59 $9^{\text {ts }}$ and sec12-4ts mutants; Figure S3: Analysis of yeast $k t i 12$ cross-complementation by ELO4 plant homologs; Figure S4: Hydropathy plots of Val-82 to Lys-102 spanning regions in Kti12 and Kti12-8; Table S1: Yeast strains; Table S2: Primers; Table S3: Plasmids.

Acknowledgments: We thank Daniel Jablonowski, Sabine Schewtschik, Christian Bär and Cindy Brachmann for help and technical advice. R.S. acknowledges grants from Deutsche Forschungsgemeinschaft (DFG: SCHA 750/18-1) and University of Kassel, Germany, Zentrale Forschungsförderung (ZFF: PhosMOrg). In addition, DFG supports by Priority Program 1784 Chemical Biology of Native Nucleic Acid Modifications to R.S. (SCHA 750/20-1), SFB648 Molecular Mechanisms of Information Processing in Plants project B to K.D.B. and R.S., an OPUS10 grant from the National Science Centre (UMO-2015/19/B/NZ1/00343) to R.K. and S.G., a grant from National Research Foundation of Korea to G-T.K. (2017R1A2B4012714), a joint-grant from BBSRC (Biotechnology and Biological Sciences Research Council, UK) to R.S (BB/F019106/1) and M.J.R.S (BB/F0191629/1) and OAP funds from the University of Kassel are greatly acknowledged.

Author Contributions: K.D.B., S.G., G.-T.K., M.J.R.S., and R.S. conceived and designed the experiments; C.M., M.W., A.H., W.A.-F., H.P., S.E.J. and R.K. performed the experiments; C.M., A.H., W.A.-F., H.P., R.K., S.G., G.-T.K., K.D.B., M.J.R.S., and R.S. analyzed the data; K.D.B., G.-T.K., M.J.R.S, and S.G. contributed materials and analysis tools; and K.D.B., S.G., M.J.R.S. and R.S. wrote the paper. 
Conflicts of Interest: The authors declare no conflict of interest.

\section{References}

1. Stark, M.J.; Boyd, A.; Mileham, A.J.; Romanos, M.A. The plasmid-encoded killer system of Kluyveromyces lactis: A review. Yeast 1990, 6, 1-29. [CrossRef] [PubMed]

2. Jablonowski, D.; Schaffrath, R. Zymocin, a composite chitinase and tRNase killer toxin from yeast. Biochem. Soc. Trans. 2007, 35, 1533-1537. [CrossRef] [PubMed]

3. Butler, A.R.; Porter, M.; Stark, M.J. Intracellular expression of Kluyveromyces lactis toxin gamma subunit mimcs treatment with exogenous toxin and distinguishes two classes of toxin resistant mutant. Yeast 1991, 7, 617-625. [CrossRef] [PubMed]

4. Kishida, M.; Tokunaga, M.; Katayose, Y.; Yajima, H.; Kawamura-Watabe, A.; Hishinuma, F. Isolation and genetic characterization of pGKL killer-insensitive mutants (iki) from Saccharomyces cerevisiae. Biosci. Biotechnol. Biochem. 1996, 60, 798-801. [CrossRef] [PubMed]

5. Butler, A.R.; White, J.H.; Folawiyo, Y.; Edlin, A.; Gardiner, D.; Stark, M.J. Two Saccharomyces cerevisiae genes which control sensitivity to G1 arrest induced by Kluyveromyces lactis toxin. Mol. Cell. Biol. 1994, 14, 6306-6316. [CrossRef] [PubMed]

6. Frohloff, F.; Fichtner, L.; Jablonowski, D.; Breunig, K.D.; Schaffrath, R. Saccharomyces cerevisiae Elongator mutations confer resistance to the Kluyveromyces lactis zymocin. EMBO J. 2001, 20, 1993-2003. [CrossRef] [PubMed]

7. Otero, G.; Fellows, J.; Li, Y.; de Bizemont, T.; Dirac, A.M.; Gustafsson, C.M.; Erdjument-Bromage, H.; Tempst, P.; Svejstrup, J.Q. Elongator, a multisubunit component of a novel RNA polymerase II holoenzyme for transcriptional elongation. Mol. Cell 1999, 3, 109-118. [CrossRef]

8. Huang, B.; Johansson, M.J.; Bystrom, A.S. An early step in wobble uridine tRNA modification requires the Elongator complex. RNA 2005, 11, 424-436. [CrossRef] [PubMed]

9. Mehlgarten, C.; Jablonowski, D.; Wrackmeyer, U.; Tschitschmann, S.; Sondermann, D.; Jäger, G.; Gong, Z.; Byström, A.S.; Schaffrath, R.; Breunig, K.D. Elongator function in tRNA wobble uridine modification is conserved between yeast and plants. Mol. Microbiol. 2010, 75, 1082-1094. [CrossRef] [PubMed]

10. Di Santo, R.; Bandau, S.; Stark, M.J. A conserved and essential basic region mediates tRNA binding to the Elp1 subunit of the Saccharomyces cerevisiae Elongator complex. Mol. Microbiol. 2014, 92, 1227-1242. [CrossRef] [PubMed]

11. Glatt, S.; Letoquart, J.; Faux, C.; Taylor, N.M.; Seraphin, B.; Müller, C.W. The Elongator subcomplex Elp456 is a hexameric RecA-like ATPase. Nat. Struct. Mol. Biol. 2012, 19, 314-320. [CrossRef] [PubMed]

12. Glatt, S.; Zabel, R.; Kolaj-Robin, O.; Onuma, O.F.; Baudin, F.; Graziadei, A.; Taverniti, V.; Lin, T.Y.; Baymann, F.; Séraphin, B.; et al. Structural basis for tRNA modification by Elp3 from Dehalococcoides mccartyi. Nat. Struct. Mol. Biol. 2016, 23, 794-802. [CrossRef] [PubMed]

13. Dauden, M.I.; Kosinski, J.; Kolaj-Robin, O.; Desfosses, A.; Ori, A.; Faux, C.; Hoffmann, N.A.; Onuma, O.F.; Breunig, K.D.; Beck, M.; et al. Architecture of the yeast Elongator complex. EMBO Rep. 2017, 18, $264-279$. [CrossRef] [PubMed]

14. Setiaputra, D.T.; Cheng, D.T.; Lu, S.; Hansen, J.M.; Dalwadi, U.; Lam, C.H.; To, J.L.; Dong, M.Q.; Yip, C.K. Molecular architecture of the yeast Elongator complex reveals an unexpected asymmetric subunit arrangement. EMBO Rep. 2017, 18, 280-291. [CrossRef] [PubMed]

15. Lu, J.; Huang, B.; Esberg, A.; Johansson, M.J.; Byström, A.S. The Kluyveromyces lactis gamma-toxin targets tRNA anticodons. RNA 2005, 11, 1648-1654. [CrossRef] [PubMed]

16. Jablonowski, D.; Zink, S.; Mehlgarten, C.; Daum, G.; Schaffrath, R. tRNA Glu wobble uridine methylation by Trm9 identifies Elongator's key role for zymocin-induced cell death in yeast. Mol. Microbiol. 2006, 59, 677-688. [CrossRef] [PubMed]

17. Kalhor, H.R.; Clarke, S. Novel methyltransferase for modified uridine residues at the wobble position of tRNA. Mol. Cell. Biol. 2003, 23, 9283-9292. [CrossRef] [PubMed]

18. Studte, P.; Zink, S.; Jablonowski, D.; Bär, C.; von der Haar, T.; Tuite, M.F.; Schaffrath, R. tRNA and protein methylase complexes mediate zymocin toxicity in yeast. Mol. Microbiol. 2008, 69, 1266-1277. [CrossRef] [PubMed] 
19. Huang, B.; Lu, J.; Bystrom, A.S. A genome-wide screen identifies genes required for formation of the wobble nucleoside 5-methoxycarbonylmethyl-2-thiouridine in Saccharomyces cerevisiae. RNA 2008, 14, 2183-2194. [CrossRef] [PubMed]

20. Satwika, D.; Klassen, R.; Meinhardt, F. Anticodon nuclease encoding virus-like elements in yeast. Appl. Microbiol. Biotechnol. 2012, 96, 345-356. [CrossRef] [PubMed]

21. Ogawa, T. tRNA-targeting ribonucleases: Molecular mechanisms and insights into their physiological roles. Biosci. Biotechnol. Biochem. 2016, 80, 1037-1045. [CrossRef] [PubMed]

22. Nandakumar, J.; Schwer, B.; Schaffrath, R.; Shuman, S. RNA repair therapy: An antidote to cytotoxic eukaryal RNA damage. Mol. Cell 2008, 31, 278-286. [CrossRef] [PubMed]

23. Fichtner, L.; Schaffrath, R. KTI11 and KTI13, Saccharomyces cerevisiae genes controlling sensitivity to G1 arrest induced by Kluyveromyces lactis zymocin. Mol. Microbiol. 2002, 44, 865-875. [CrossRef] [PubMed]

24. Fichtner, L.; Frohloff, F.; Bürkner, K.; Larsen, M.; Breunig, K.D.; Schaffrath, R. Molecular analysis of KTI12/TOT4, a Saccharomyces cerevisiae gene required for Kluyveromyces lactis zymocin action. Mol. Microbiol. 2002, 43, 783-791. [CrossRef] [PubMed]

25. Mehlgarten, C.; Schaffrath, R. Mutant casein kinase I (Hrr25p/Kti14p) abrogates the G1 cell cycle arrest induced by Kluyveromyces lactis zymocin in budding yeast. Mol. Genet. Genom. 2003, 269, 188-196.

26. Jablonowski, D.; Fichtner, L.; Stark, M.J.R.; Schaffrath, R. The yeast Elongator histone acetylase requires Sit4-dependent dephosphorylation for toxin-target capacity. Mol. Biol. Cell 2004, 15, 1459-1469. [CrossRef] [PubMed]

27. Jablonowski, D.; Täubert, J.-E.; Bär, C.; Stark, M.J.R.; Schaffrath, R. Distinct subsets of Sit4 holo-phosphatases are required for inhibition of yeast growth by rapamycin and zymocin. Eukaryot. Cell 2009, 8, 1637-1647. [CrossRef] [PubMed]

28. Mehlgarten, C.; Jablonowski, D.; Breunig, K.D.; Stark, M.J.R.; Schaffrath, R. Elongator function depends on antagonistic regulation by casein kinase Hrr25 and protein phosphatase Sit4. Mol. Microbiol. 2009, 73, 869-881. [CrossRef] [PubMed]

29. Abdel-Fattah, W.; Jablonowski, D.; Di Santo, R.; Scheidt, V.; Hammermeister, A.; ten Have, S.M.; Thüring, K.L.; Helm, M.; Schaffrath, R.; Stark, M.J.R. Phosphorylation of Elp1 by Hrr25 is required for Elongator-dependent tRNA modification in yeast. PLoS Genet. 2015, 11, e1004931. [CrossRef] [PubMed]

30. Leipe, D.D.; Koonin, E.V.; Aravind, L. Evolution and classification of P-loop kinases and related proteins. J. Mol. Biol. 2003, 333, 781-815. [CrossRef] [PubMed]

31. Jun, S.E.; Cho, K.-H.; Hwang, J.-Y.; Abdel-Fattah, W.; Hammermeister, A.; Schaffrath, R.; Bowman, J.L.; Kim, G.T. Comparative analysis of the conserved functions of Arabidopsis DRL1 and yeast KTI12. Mol. Cells 2015, 38, 243-250. [CrossRef] [PubMed]

32. Nelissen, H.; Clarke, J.H.; De Block, M.; De Block, S.; Vanderhaeghen, R.; Zielinski, R.E.; Dyer, T.; Lust, S.; Inzé, D.; Van Lijsebettens, M. DRL1, a homolog of the yeast TOT4/KTI12 protein, has a function in meristem activity and organ growth in plants. Plant Cell 2003, 15, 639-654. [CrossRef] [PubMed]

33. Nelissen, H.; Fleury, D.; Bruno, L.; Robles, P.; De Veylder, L.; Traas, J.; Micol, J.L.; Van Montagu, M.; Inzé, D.; Van Lijsebettens, M. The elongata mutants identify a functional Elongator complex in plants with a role in cell proliferation during organ growth. Proc. Natl. Acad. Sci. USA 2005, 102, 7754-7759. [CrossRef] [PubMed]

34. Puig, O.; Caspary, F.; Rigaut, G.; Rutz, B.; Bouveret, E.; Bragado-Nilsson, E.; Wilm, M.; Séraphin, B. The tandem affinity purification (TAP) method: A general procedure of protein complex purification. Methods 2001, 24, 218-229. [CrossRef] [PubMed]

35. Rhoads, A.R.; Friedberg, F. Sequence motifs for calmodulin recognition. FASEB J. 1997, 11, 331-340. [PubMed]

36. Petrakis, T.G.; Søgaard, T.M.; Erdjument-Bromage, H.; Tempst, P.; Svejstrup, J.Q. Physical and functional interaction between Elongator and the chromatin-associated Kti12 protein. J. Biol. Chem. 2005, 280, 19454-19460. [CrossRef] [PubMed]

37. Klassen, R.; Ciftci, A.; Funk, J.; Bruch, A.; Butter, F.; Schaffrath, R. tRNA anticodon loop modifications ensure protein homeostasis and cell morphogenesis in yeast. Nucleic Acids Res. 2016, 44, 10946-10959. [CrossRef] [PubMed]

38. Rahl, P.B.; Chen, C.Z.; Collins, R.N. Elp1p, the yeast homolog of the FD disease syndrome protein, negatively regulates exocytosis independently of transcriptional elongation. Mol. Cell 2005, 17, 841-853. [CrossRef] [PubMed] 
39. Zabel, R.; Bär, C.; Mehlgarten, C.; Schaffrath, R. Yeast -tubulin suppressor Ats1/Kti13 relates to the Elongator complex and interacts with Elongator partner protein Kti11. Mol. Microbiol. 2008, 69, 175-187. [CrossRef] [PubMed]

40. Johansson, M.J.; Esberg, A.; Huang, B.; Bjork, G.R.; Bystrom, A.S. Eukaryotic wobble uridine modifications promote a functionally redundant decoding system. Mol. Cell. Biol. 2008, 28, 3301-3312. [CrossRef] [PubMed]

41. Rezgui, V.A.; Tyagi, K.; Ranjan, N.; Konevega, A.L.; Mittelstaet, J.; Rodnina, M.V.; Peter, M.; Pedrioli, P.G. tRNA $\mathrm{tK}_{\mathrm{UUU}}, \mathrm{tQUUG}$, and $\mathrm{tE} \mathrm{UUC}_{\mathrm{U}}$ wobble position modifications fine-tune protein translation by promoting ribosome A-site binding. Proc. Natl. Acad. Sci. USA 2013, 110, 12289-12294. [CrossRef] [PubMed]

42. Nedialkova, D.D.; Leidel, S.A. Optimization of codon translation rates via tRNA modifications maintains proteome inegrity. Cell 2015, 161, 1606-1618. [CrossRef] [PubMed]

43. Ranjan, N.; Rodnina, M.V. Thio-modification of tRNA at the wobble position as regulator of the kinetics of decoding and translocation on the ribosome. J. Am. Chem. Soc. 2017, 139, 5857-5864. [CrossRef] [PubMed]

44. Tükenmez, H.; Xu, H.; Esberg, A.; Byström, A.S. The role of wobble uridine modifications in +1 translational frameshifting in eukaryotes. Nucleic Acids Res. 2015, 43, 9489-9499. [CrossRef] [PubMed]

45. Klassen, R.; Bruch, A.; Schaffrath, R. Independent suppression of ribosomal +1 frameshifts by different $t$ RNA anticodon loop modifications. RNA Biol. 2017. [CrossRef] [PubMed]

46. Karlsborn, T.; Tükenmez, H.; Mahmud, A.K.; Xu, F.; Xu, H.; Byström, A.S. Elongator, a conserved complex required for wobble uridine modifications in eukaryotes. RNA Biol. 2014, 11, 1519-1528. [CrossRef] [PubMed]

47. Schaffrath, R.; Leidel, S.A. Wobble uridine modifications-A reason to live, a reason to die?! RNA Biol. 2017. [CrossRef] [PubMed]

48. Klassen, R.; Grunewald, P.; Thüring, K.L.; Eichler, C.; Helm, M.; Schaffrath, R. Loss of anticodon wobble uridine modifications affects tRNA ${ }^{\text {Lys }}$ function and protein levels in Saccharomyces cerevisiae. PLoS ONE 2015, 11, e0119261. [CrossRef] [PubMed]

49. Jüdes, A.; Bruch, A.; Klassen, R.; Helm, M.; Schaffrath, R. Sulfur transfer and activation by ubiquitin-like modifier system Uba4•Urm1 link protein urmylation and tRNA thiolation in yeast. Microb. Cell 2016, 3, 423-433. [CrossRef] [PubMed]

50. Su, J.Y.; Belmont, L.; Sclafani, R.A. Genetic and molecular analysis of the SOE1 gene: A tRNA (3Glu) missense suppressor of yeast $c d c 8$ mutations. Genetics 1990, 124, 523-531. [PubMed]

51. Chen, Z.; Zhang, H.; Jablonowski, D.; Zhou, X.; Ren, X.; Hong, X.; Schaffrath, R.; Zhu, J.-K.; Gong, Z. Mutations in ABO1/ELO2, one subunit of holo-Elongator, increase ABA sensitivity and drought tolerance in Arabidopsis. Mol. Cell. Biol. 2006, 26, 6902-6912. [CrossRef] [PubMed]

52. Bär, C.; Zabel, R.; Liu, S.; Stark, M.J.; Schaffrath, R. A versatile partner of eukaryotic protein complexes that is involved in multiple biological processes: Kti11/Dph3. Mol. Microbiol. 2008, 69, 1221-1233. [PubMed]

53. Glatt, S.; Müller, C.W. Structural insights into Elongator function. Curr. Opin. Struct. Biol. 2013, 23, $235-242$. [CrossRef] [PubMed]

54. Glatt, S.; Zabel, R.; Vonkova, I.; Kumar, A.; Netz, D.J.; Pierik, A.J.; Rybin, V.; Lill, R.; Gavin, A.C.; Balbach, J.; et al. Structure of the Kti11/Kti13 heterodimer and its double role in modification of tRNA and eukaryotic elongation factor 2. Structure 2015, 23, 149-160. [CrossRef] [PubMed]

55. Kolaj-Robin, O.; McEwen, A.G.; Cavarelli, J.; Séraphin, B. Structure of the Elongator cofactor complex Kti11/Kti13 provides insight into the role of Kti13 in Elongator-dependent tRNA modification. FEBS J. 2015, 282, 819-833. [CrossRef] [PubMed]

56. Karlsborn, T.; Mahmud, A.K.M.F.; Tükenmez, H.; Byström, A.S. Loss of $\mathrm{ncm}^{5}$ and $\mathrm{mcm}^{5}$ wobble uridine side chains results in an altered metabolic profile. Metabolomics 2016, 12, 177. [CrossRef] [PubMed]

57. Sherman, F. Guide to yeast genetics and molecular biology. Getting started with yeast. Methods Enzymol. 1991, 194, 3-20. [PubMed]

58. Gietz, R.D.; Woods, R.A. Transformation of yeast by lithium acetate/single-stranded carrier DNA/polyethylene glycol method. Methods Enzymol. 2002, 350, 87-96. [PubMed]

59. Gietz, R.D.; Sugino, A. New yeast-Escherichia coli shuttle vectors constructed with in vitro mutagenized yeast genes lacking six-base pair restriction sites. Gene 1988, 74, 527-534. [CrossRef] 
60. Kelley, L.A.; Mezulis, S.; Yates, C.M.; Wass, M.N.; Sternberg, M.J.; Kelley, L.A.; Mezulis, S.; Yates, C.M.; Wass, M.N.; Sternberg, M.J. The Phyre2 web portal for protein modeling, prediction and analysis. Nat. Protoc. 2015, 10, 845-858. [CrossRef] [PubMed]

61. Sherrer, R.L.; O'Donoghue, P.; Söll, D. Characterization and evolutionary history of an archaeal kinase involved in selenocysteinyl-tRNA formation. Nucleic Acids Res. 2008, 36, 1247-1259. [CrossRef] [PubMed]

62. Waterhouse, A.M.; Procter, J.B.; Martin, D.M.A.; Clamp, M.; Barton, G.J. Jalview Version 2-a multiple sequence alignment editor and analysis workbench. Bioinformatics 2009, 25, 1189-1191. [CrossRef] [PubMed]

63. Celniker, G.; Nimrod, G.; Ashkenazy, H.; Glaser, F.; Martz, E.; Mayrose, I.; Pupko, T.; Ben-Tal, N. ConSurf: Using Evolutionary Data to Raise Testable Hypotheses about Protein Function. Isr. J. Chem. 2013, 53, $199-206$. [CrossRef]

(C) 2017 by the authors. Licensee MDPI, Basel, Switzerland. This article is an open access article distributed under the terms and conditions of the Creative Commons Attribution (CC BY) license (http:/ / creativecommons.org/licenses/by/4.0/). 